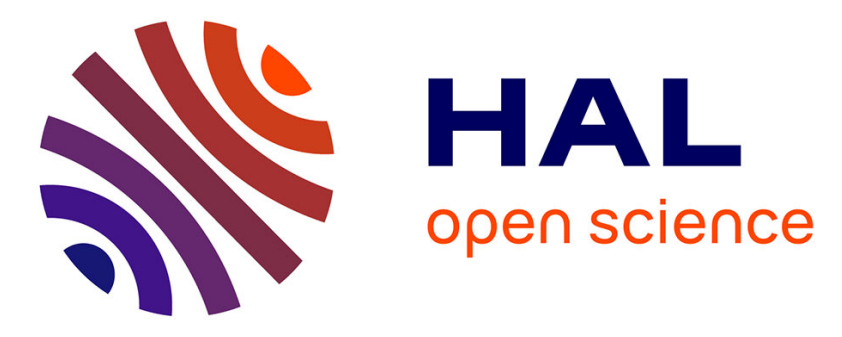

\title{
Analysis of the application of optical two wavelength techniques to measurement of the Soret coefficients in ternary mixtures.
}

Valentina Shevtsova, Vitaliy Sechenyh, Alexander Nepomnyashchy, Jean

Claude Legros

\section{To cite this version:}

Valentina Shevtsova, Vitaliy Sechenyh, Alexander Nepomnyashchy, Jean Claude Legros. Analysis of the application of optical two wavelength techniques to measurement of the Soret coefficients in ternary mixtures.. Philosophical Magazine, 2011, pp.1. 10.1080/14786435.2011.586376 . hal-00710062

\section{HAL Id: hal-00710062 \\ https://hal.science/hal-00710062}

Submitted on 20 Jun 2012

HAL is a multi-disciplinary open access archive for the deposit and dissemination of scientific research documents, whether they are published or not. The documents may come from teaching and research institutions in France or abroad, or from public or private research centers.
L'archive ouverte pluridisciplinaire HAL, est destinée au dépôt et à la diffusion de documents scientifiques de niveau recherche, publiés ou non, émanant des établissements d'enseignement et de recherche français ou étrangers, des laboratoires publics ou privés. 


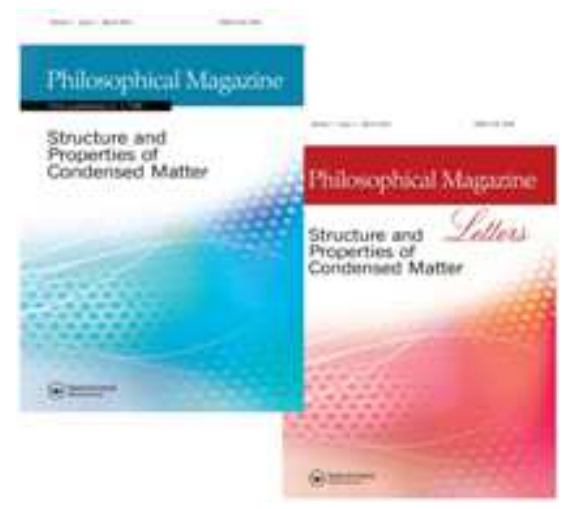

\section{Analysis of the application of optical two wavelength techniques to measurement of the Soret coefficients in ternary mixtures.}

\begin{tabular}{|c|c|}
\hline Journal: & Philosophical Magazine \& Philosophical Magazine Letters \\
\hline Manuscript ID: & TPHM-11-Feb-0084 \\
\hline Journal Selection: & Philosophical Magazine \\
\hline $\begin{array}{r}\text { Date Submitted by the } \\
\text { Author: }\end{array}$ & 28-Feb-2011 \\
\hline Complete List of Authors: & $\begin{array}{l}\text { Shevtsova, Valentina; University of Brussels (ULB), Chemical } \\
\text { Physics, EP - CP165/62 } \\
\text { Sechenyh, Vitaliy; University of Brussels (ULB), Chemical Physics, } \\
\text { EP - CP165/62 } \\
\text { Nepomnyashchy, Alexander; University of Brussels (ULB), Chemical } \\
\text { Physics, EP - CP165/62 } \\
\text { Legros, Jean Claude; University of Brussels (ULB), Chemical } \\
\text { Physics, EP - CP165/62 }\end{array}$ \\
\hline Keywords: & diffusion, Soret effect, refractive index, liquids \\
\hline Keywords (user supplied): & \\
\hline
\end{tabular}

\section{SCHOLARONE ${ }^{\text {M }}$ \\ Manuscripts}


Philosophical Magazine

Vol. 00, No. 00, 23 February 2011, 1-18

\title{
RESEARCH ARTICLE
}

\section{Analysis of the application of optical two wavelength techniques to measurement of the Soret coefficients in ternary mixtures}

\author{
V. Shevtsova*, V. Sechenyh, A. Nepomnyashchy and J.C. Legros \\ MRC, CP-165/62, Université Libre de Bruxelles, 50, av. F.D.Roosevelt, B-1050 \\ Brussels, Belgium \\ (Received 00 Month 200x; final version received 00 Month 200x)
}

\begin{abstract}
We discuss the application of two-wavelength optical methods for the determination of Soret coefficient in ternary mixtures. Though the principle of such an approach was formulated in the literature earlier, we have found a significant obstacle to its implementation and suggested a way to circumvent that obstacle. The main target of our approach is the analysis of the compatibility of the matrices of contrast factors (derivatives of refractive indices with respect to concentrations) with available light sources used for measuring the Soret effect in ternary mixtures. We report on the development of a simple mathematical approach to verify applicability of chosen wave lengths for laser diodes to measure transport coefficients for a certain class of ternary mixtures. The approach has been applied to a number of aqueous ternary mixtures: Water-Ethanol-k-Ethyleneglycol, $\mathrm{k}=$ mono, di, tri and Water-t-butanolDMSO) and light sources in visible $\lambda_{1}=670 \mathrm{~nm}$ and infrared $\lambda_{2}=925 \mathrm{~nm}$ spectrum. The regions of feasibility/infeasibility of the methods have been revealed for these mixtures.
\end{abstract}

Keywords: diffusion; Soret effect; refractive index; condition number

\section{Introduction}

The liquids appearing in nature and industrial applications are essentially multicomponent. The behavior of multicomponent systems is more complicated in comparison with pure fluids due to a complex interplay between heat and mass transfer processes [1]. Specifically, the diffusive mass transport of a given component is induced not only by its compositional gradient (main or principal diffusion), but also by the compositional gradients of the other components (cross-diffusion) and the temperature gradient (thermodiffusion, also called Soret effect). The prediction of mass transfer processes in multicomponent systems greatly relies on the knowledge of diffusion and thermodiffusion coefficients, which appear in the equations describing these phenomena. The appearance of cross-molecular diffusion complicates measurement of the coefficients in ternary and higher mixtures in comparison to that in binary mixtures.

There are many important processes in nature and technology, where the abovementioned phenomena play a crucial role. The composition of hydrocarbon reservoirs is significantly affected by diffusion as well as by the Soret effect (due to the presence of geo-thermal gradient) [2], [3]. The thermodiffusion is employed for isotope separation in liquid and gaseous mixtures as well as in other separation

*Corresponding author. Email: vshev@ulb.ac.be

ISSN: 1478-6435 print/ISSN 1478-6443 online 
processes [4] that involve colloids, macromolecules or nano-fluids. Other potential applications include high-pressure combustion [5], solidification processes [6], oceanic convection [8], biological systems [9] and more recently appeared problems linked to $\mathrm{CO}_{2}$ geological storage [7].

Different formalisms and theories exist in literature to describe the thermodiffusion effect in multicomponent mixtures. Firoozabadi et al. [10] developed a model using irreversible thermodynamics which focuses on connecting the heat of transport with liquid viscosity activation energy. Closure of the model requires mixing rules and/or some constants, among which equilibrium properties (partial molar properties) are obtained from the Peng-Robinson equation of state, while nonequilibrium properties are determined using the energy of viscous flow estimated from viscosity data. Another model [11] for multicomponent mixtures uses statistical thermodynamic methods when the Soret coefficient consists of two contributions, a kinetic one and a thermodynamic one. The model is based on the maximization of the partition function of two large, uniform but differently heated idealized "bulbs" connected by a small tube with a linear temperature profile. The authors emphasize that the theoretically determined Soret coefficient is extremely sensitive to the used equation of state.

The results of the model [10] were favorably compared with a single available experimental spatial concentration data [12]. At that time, there were no experimental data for multicomponent thermal diffusion factors. The new and accurate experimental data will motivate further work to understand the process of thermal diffusion from a theoretical point of view. The long-term vision is to understand thermal diffusion in multicomponent systems. Ternary systems are merely the first step in this direction. But even this step is rather complicated and ternaries are already much different from binaries.

Recently, the first experimental data have appeared for the thermodiffusion coefficients which can be used for testing the models in ternary mixtures. Kita et al. [13] studied a quasi-ternary mixture by an optical grating technique. "Quasiternary" means that their analysis is based on two assumptions: (1) the off-diagonal diffusion coefficients are negligible, and (2) the diagonal diffusion coefficients are of different orders of magnitude.

Until now the values of Soret coefficient were measured only in three hydrocarbon liquid ternary mixtures: (\#1) $n C_{12} H_{26}-T H N-I B B$ (dodecanetetrahydronaphthalenene-isobutylbenzene); (\#2) $n C_{10} H_{22}-T H N-I B B$ (ndecane instead dodecane); (\#3) $n C_{8}-n C_{10}-M N$ (mixture of alkanes and 1methylnaphthalene). The binary contributions of mixture \#1 were used as benchmark liquids [14], and it appears that this mixture also plays the role of a benchmark mixture among the ternary ones.

Using the thermogravitational column technique, Leahy-Dios et al. [15] measured the thermodiffusion coefficients in mixtures \#1 and \#3 (for two compositions). Using a plane-thermogravitational column with a small gap dimension, Blanco et al. [16] measured the thermodiffusion coefficients in mixture \#1 (four compositions) and \#2 (two compositions) with a goal to improve the accuracy of the results reported in [15]. By using beam deflection technique Königer et al. [17] measured the Soret coefficient in mixtures \#1 and \#3. They reported only partial agreement with the previous measurements [16].

Until now the validity of the additive rule to estimate the thermodiffusion coefficients in a ternary mixture from those in the corresponding binary mixtures at the same mass fraction ratio (so-called mixing rule) is under discussion. The authors of [16] claim that this rule holds satisfactorily for the studied hydrocarbon mixtures $\# 1$ and \#2 while the authors of [15] indicate that for mixture \#3 this rule does 


\section{$\begin{array}{rrr}\text { February 28, 2011 } & 17: 40 & \text { Philosophical Magazine } \\ \text { Page } \mathbf{3} \text { of } \mathbf{1 8} & \text { Philosophical Magazine \& Philosophical Magazine Letters }\end{array}$}

Philosophical Magazine

not hold. So, there is a real need to conduct measurements of the Soret effect in ternary mixtures.

Taking into account that the Soret coefficients obtained in [16] and [17] differ not only in values but even in signs, the need in trustworthy results is obvious. One of the ways for solving this problem is to conduct an experiment in the absence of gravity on the ISS, with a purpose to provide incontestable benchmark results for ground experiments. Such an experiment should be carefully prepared keeping in mind that the facility available currently on the ISS is the SODI instrument. That instrument includes an optical interferometer, and its real time results (images) can be sent to researchers via telemetry.

Among the existing methods for measurement of thermodiffusion coefficients in binary mixtures, the modern optical techniques play an important role - such as Thermal Lens Technique [18], [19], Beam Deflection [17, 20], Thermal Diffusion Forced Rayleigh Scattering [21, 22], Optical Digital Interferometry [23, 24]. They require accurate knowledge of refractive index derivatives (contrast factor) with respect to concentration and temperature.

Moving to ternary mixtures the success of the experiments depends not only on contrast factors of individual components but on the matrix composed by $\partial n_{\lambda_{j}} / \partial c_{i}, i, j=1,2$. There are two equivalent ways to choose optimal experimental conditions: by choosing a concentration set or by choosing a proper wave length. In the case of interest for some specific liquids one should search for proper light sources while for the prescribed wavelengths not all compositions are suitable. To our knowledge, the present paper is one of the first studies with the goal to find the proper approach for determination of compatibility between the compositions and the wavelengths. As a distinctive example we have chosen the wavelengths $\lambda_{1}=670 \mathrm{~nm}$ and $\lambda_{2}=925 \mathrm{~nm}$ available on the ISS in the SODI instrument and examine different mixtures.

The purpose of the current paper is two-fold: (a) to present a theoretical approach for the best choice of compositions inside mixtures, which can be accurately measured by two-wavelength technique; (b) to present extensive measurements of contrast factors in ternary aqueous solutions (water-ethanol-k-ethyleneglycol, $\mathrm{k}=$ mono, di, tri) and water-t-butanol-DMSO.

\section{Theoretical background}

\subsection{Mass fluxes}

We consider a ternary mixture, where two concentrations 1 and 2 are chosen as independent. If we denote the mass fraction of component $i$ by $C_{i}$, then $C_{1}+C_{2}+$ $C_{3}=1$ and the diffusive fluxes of independent components can be written as

$$
\begin{aligned}
& J_{1}=-\rho_{0} D_{11} \nabla C_{1}-\rho_{0} D_{12} \nabla C_{2}-\rho_{0} D_{T 1}^{\prime} \nabla T, \\
& J_{2}=-\rho_{0} D_{21} \nabla C_{1}-\rho_{0} D_{22} \nabla C_{2}-\rho_{0} D_{T 2}^{\prime} \nabla T,
\end{aligned}
$$

where $D_{i j}$ are the mass-based diffusion coefficients and $D_{T i}^{\prime}$ are the thermal diffusion coefficients. The transport coefficients are supposed to be constant in a sufficiently small range of temperature and concentration variations and correspond to the mean temperature $T_{0}$ and composition $C_{0}=\left(C_{10}, C_{20}\right)$. The diffusive flux $J_{3}$ is defined from the condition that the fluxes of all components must sum up to zero. Since the diffusive flux of component $i$ must vanish at the limits $C_{i 0}=0$ and 
$C_{i 0}=1$, the thermal diffusion coefficients can be represented as [25]-[27]

$$
D_{T i}^{\prime}=C_{i 0}\left(1-C_{i 0}\right) D_{T i}
$$

to underline zeros of function $D_{T i}^{\prime}$. This relation can mask other essential dependences of $D_{T i}^{\prime}$ on the concentrations. For instance, if we take the limit $C_{1} \rightarrow 0$ while keeping $C_{2}$ and $C_{3}$ between 0 and 1 , then the fluxes $J_{2}$ and $J_{3}$ proportional to $\nabla T$ will be nonzero, while $J_{1}=-J_{2}-J_{3}$ has to tend to zero. Therefore, the dependences of $D_{T 2}$ and $D_{T 3}$ on the concentration have to be rather nontrivial: it should be $D_{T 2}^{\prime}+D_{T 3}^{\prime} \rightarrow 0$ when $C_{2}+C_{3} \rightarrow 1$. So, the actual dependence of $D_{T i}^{\prime}$ on concentrations is more tricky than just the formula that we have presented, i.e. $D_{T i}$ still depend on all the concentrations.

In laboratory experiments a nonuniform temperature field is created in the cell by maintaining a steady temperature difference between the boundaries, e.g. by heating the upper wall and cooling the bottom wall. In the absence of motion, the system reaches a stationary state, which is described by the condition of zero net diffusive flux in the system:

$$
\begin{gathered}
D_{11} \nabla C_{1}+D_{12} \nabla C_{2}+D_{T 1}^{\prime} \nabla T=0 \\
D_{21} \nabla C_{1}+D_{22} \nabla C_{2}+D_{T 2}^{\prime} \nabla T=0
\end{gathered}
$$

So, the applied temperature gradient creates the stationary concentration gradient due to thermal diffusion. The Soret coefficients $S_{T 1}, S_{T 2}$ of components are defined by relations $[29]$

$$
S_{T 1} \nabla T \equiv-\frac{1}{C_{10}\left(1-C_{10}\right)} \nabla C_{1}, \quad S_{T 2} \nabla T \equiv-\frac{1}{C_{20}\left(1-C_{20}\right)} \nabla C_{2},
$$

and from the solution of Eqs.(5)-(4) it follows

$$
\nabla C_{1}=-\frac{D_{T 1}^{\prime} D_{22}-D_{T 2}^{\prime} D_{12}}{D_{11} D_{22}-D_{12} D_{21}} \quad \nabla T, \quad \nabla C_{2}=-\frac{D_{T 2}^{\prime} D_{11}-D_{T 1}^{\prime} D_{21}}{D_{11} D_{22}-D_{12} D_{21}} \quad \nabla T .
$$

Consequently, the Soret coefficients can be determined as

$$
\begin{array}{r}
S_{T 1}=-\frac{D_{T 1} D_{22}-R_{0} D_{T 2} D_{12}}{D_{11} D_{22}-D_{12} D_{21}}, \quad S_{T 2}=-\frac{D_{T 2} D_{11}-R_{0}^{-1} D_{T 1} D_{21}}{D_{11} D_{22}-D_{12} D_{21}}, \\
\text { here } \quad R_{0}=C_{20}\left(1-C_{20}\right) / C_{10}\left(1-C_{10}\right)>0 .
\end{array}
$$

It follows from Eqs. (7) that the signs of the Soret coefficients depend not only on $D_{T i}$ but also can be affected by the diffusion and cross-diffusion coefficients.

The described relations provide a basis for a number of methods for measuring thermal diffusion coefficients in a steady state and, particularly, for the above-mentioned OBD and ODI. The transient approach was considered by Haugen\&Firoozabadi [30].

In both optical methods, beam deflection [17], [31] and digital interferometry [24], [32], the temperature gradient is imposed between horizontal walls. Due to thermal diffusion, the components of the mixture start to segregate and variations of the refractive index due to temperature and concentration non-uniformity are recorded. One may find the detailed description of the working principle in the corresponding references.

Since two independent concentrations should be measured in ternary mixture, two laser diodes emitting different wave lengths that ensure a different optical be- 


\section{Page 5 of 18

havior of the components, are required for a complete composition analysis. The beam deflection technique has been already applied for studying ternary mixtures [17], while application of digital interferometry was reported until now only for binary mixtures [24], and its application for ternary ones is foreseen in the forthcoming microgravity experiments.

\subsection{Working equations}

For a given wavelength $\lambda$, the variation of the refractive index $n(x, z)$ includes temperature and concentration contributions

$$
\Delta n(x, z)=\left(\frac{\partial n}{\partial C_{1}}\right)_{T_{0}, C_{20}} \Delta C_{1}(x, z)+\left(\frac{\partial n}{\partial C_{2}}\right)_{T_{0}, C_{10}} \Delta C_{2}(x, z)+\left(\frac{\partial n}{\partial T}\right)_{C_{10}, C_{20}} \Delta T(x, z),
$$

where $\Delta T(x, z)$ and $\Delta C_{1,2}(x, z)$ are temperature and concentration changes at the point $(x, z)$, here $z$-axis is in the direction of temperature gradient.

Initially, the system is in a homogeneous state, and then a temperature gradient is applied. From the images recorded early in time $\left(t=\tau_{t h}<<\tau_{D}\right)$, one can determine temperature field $\Delta T\left(x, z, \tau_{t h}\right)=\Delta T_{\text {ref }}(x, z)$. The characteristic thermal time $\tau_{t h}=L^{2} / \chi$ is a hundred times shorter than the diffusion time $\tau_{D}=L^{2} / D$ (here $L$ is the length in the direction of the temperature gradient, $\chi$ is the thermal diffusivity, and $D$ is the characteristic scale of the diffusion coefficients). Because for $t=\tau_{t h}$ the concentration changes are still negligible,

$$
\Delta n_{i}^{T}(x, z)=\left(\frac{\partial n_{i}}{\partial T}\right)_{C_{10}, C_{20}} \Delta T_{r e f}\left(x, z, \tau_{t h}\right), i=1,2 .
$$

Later in time the refractive index will change only due to the Soret induced variations of the concentration fields

$$
\begin{aligned}
& \left(\frac{\partial n_{1}}{\partial C_{1}}\right)_{T_{0}, C_{20}} \Delta C_{1}(x, z, t)+\left(\frac{\partial n_{1}}{\partial C_{2}}\right)_{T_{0}, C_{10}} \Delta C_{2}(x, z, t)=\Delta n_{1}(x, z, t)-\Delta n_{1}^{T}(x, z)=F_{1}, \\
& \left(\frac{\partial n_{2}}{\partial C_{1}}\right)_{T_{0}, C_{20}}^{\Delta} \Delta C_{1}(x, z, t)+\left(\frac{\partial n_{2}}{\partial C_{2}}\right)_{T_{0}, C_{10}}^{\Delta} \Delta C_{2}(x, z, t)=\Delta n_{2}(x, z, t)-\Delta n_{2}^{T}(x, z)=F_{2} .
\end{aligned}
$$

Variations of refractive indices with concentrations, $\partial n_{i} / \partial C_{j}$, (the so-called contrast factors) depend on the wave length of light sources and should be accurately measured for the chosen compositions $C_{10}, C_{20}$ and $T_{0}$. Here our attention is focused on how these values and accuracy of their measurements influence the determination of $\Delta C_{i}$.

Knowing $\Delta n_{1}(x, z, t)$ and $\Delta n_{2}(x, z, t)$ from the measurements, equations (9)(10) can be written in a vector form where the matrix $A$ should be determined from preparatory laboratory experiments,

$$
A \Delta \vec{C}=\vec{F} \quad \text { where } \quad A=\left(\begin{array}{cc}
\frac{\partial n_{1}}{\partial C_{1}} & \frac{\partial n_{1}}{\partial C_{2}} \\
\frac{\partial n_{2}}{\partial C_{1}} & \frac{\partial n_{2}}{\partial C_{2}}
\end{array}\right) \quad \text { and } \quad \vec{F}=\left(\begin{array}{c}
F_{1} \\
F_{2}
\end{array}\right) .
$$

By inversion of the matrix $A$, we find the variations of the concentration fields,

$$
\Delta \vec{C}=A^{-1} \vec{F}
$$

and then we find the Soret coefficients using relations (5). 


\subsection{Condition number}

The condition number $K$ of the matrix gives an estimate from above for a ratio of the relative error of a definite quantity ("norm") characterizing the calculated vector $\left(\Delta C_{1}, \Delta C_{2}\right)$ to the relative error characterizing the data vector $\left(F_{1}, F_{2}\right)$. For instance, if $K=10^{2}$, then for the data $\left(F_{1}, F_{2}\right)$ measured with the relative error about $10^{-3}$, the relative error for $\left(\Delta C_{1}, \Delta C_{2}\right)$ can reach $10^{-1}$. The norms which are typically used are as follows

$$
\begin{array}{lll}
\text { (i) } & l_{1}-\text { norm } & \|\Delta C\|_{1}=\left|\Delta C_{1}\right|+\left|\Delta C_{2}\right|, \\
\text { (ii) } & l_{2}-\text { norm } & \|\Delta C\|_{2}=\sqrt{\left|\Delta C_{1}\right|^{2}+\left|\Delta C_{2}\right|^{2}}, \\
\text { (iii) } & l_{\infty}-\text { norm } & \|\Delta C\|_{\infty}=\max \left(\left|\Delta C_{1}\right|,\left|\Delta C_{2}\right|\right) .
\end{array}
$$

The condition numbers calculated using different norms do not exactly coincide.

Note that using the relation $\Delta C_{1}+\Delta C_{2}+\Delta C_{3}=0$, we can transform (11) into equivalent pairs of equations for $\left(\Delta C_{2}, \Delta C_{3}\right)$ or $\left(\Delta C_{1}, \Delta C_{3}\right)$. Hence, on the basis 


\section{bruary 28, 2011

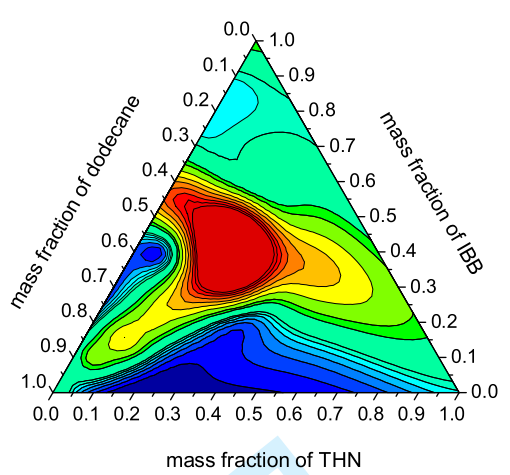

(a)

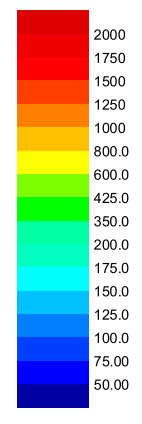

a)

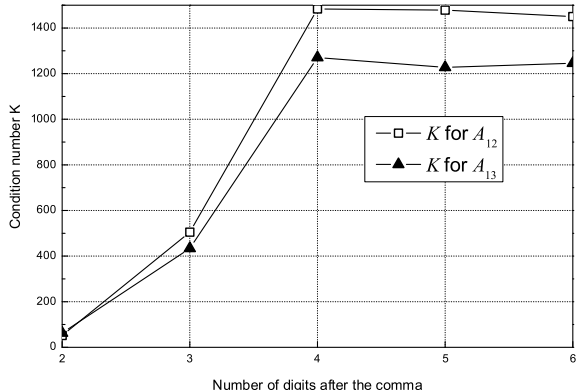

(b)

Figure 2. (a) Isolines of the condition number $K\left(C_{10}, C_{30}\right)$ of matrix $A_{13}$. Darker regions correspond to smaller $K$. b) The dependence of the condition numbers of the matrices $A_{12}$ and $A_{13}$ and on the number of digits after comma for matrix elements.

of the measured $n$ and the determined derivatives one can construct three matrices

$$
A_{12}=\left(\begin{array}{ll}
\frac{\partial n_{1}}{\partial C_{1}} & \frac{\partial n_{1}}{\partial C_{2}} \\
\frac{\partial n_{2}}{\partial C_{1}} & \frac{\partial n_{2}}{\partial C_{2}}
\end{array}\right) \quad A_{23}=\left(\begin{array}{ll}
\frac{\partial n_{1}}{\partial C_{2}} & \frac{\partial n_{1}}{\partial C_{3}} \\
\frac{\partial n_{2}}{\partial C_{2}} & \frac{\partial n_{2}}{\partial C_{3}}
\end{array}\right) \quad A_{13}=\left(\begin{array}{ll}
\frac{\partial n_{1}}{\partial C_{1}} & \frac{\partial n_{1}}{\partial C_{3}} \\
\frac{\partial n_{2}}{\partial C_{1}} & \frac{\partial n_{2}}{\partial C_{3}}
\end{array}\right)
$$

The condition numbers of these three matrices are not exactly equal, because the corresponding norms are chosen in a different way (e.g., for $A_{23},\|\Delta C\|_{1}=\left|\Delta C_{2}\right|+$ $\left|\Delta C_{3}\right|$, which is different from (12)).

It should be noted that the actual relative error of the quantities $\left|\Delta C_{1}\right|,\left|\Delta C_{2}\right|$ and $\left|\Delta C_{3}\right|$ depends not only on the relative error of the data $F_{1}, F_{2}$, but on the data themselves, and it cannot be found exactly without knowing those data. The condition numbers determine the order of magnitude of the error for quantities (12)-(14) in the worst case.

For planning an experiment, one can calculate the condition number for different pairs of light wavelengths and for different values of $\left(C_{10}, C_{20}\right)$, searching for its minimum. Note, that although the value of $K$ depends on the norm selected among Eqs.(12)-(14), the minimal and maximal values of $K\left(C_{10}, C_{20}\right)$ take place approximately at the same values of $\left(C_{10}, C_{20}\right)$ for different norms. Thus, regardless of the chosen norm one can identify the region of $K\left(C_{10}, C_{20}\right)$ where solution is the most precise. Hereafter the norm $l_{2}$ is used, as it is easy to calculate (for the details of the condition number calculation, see Appendix B).

To illustrate the application of the condition number calculation for the experiment planning, let us consider the matrix $A_{13}$ using the data obtained for $\mathrm{C}_{12} \mathrm{H}_{26}-\mathrm{IBB}-\mathrm{THN}$ mixture with the pair of wavelengths $\lambda_{1}=670 \mathrm{~nm}$ and $\lambda_{2}=925 \mathrm{~nm}$. The experimental procedure for finding the elements of the matrix is described in Sec. III. The calculated condition numbers $K\left(C_{10}, C_{30}\right)$ for the matrix $A_{13}$ are shown in Fig. 2a. The darker is the region, the better is the accuracy of solving the equations. The point of interest $C_{i 0}=\{33.334 \%-33.333 \%-33.333 \%\}$ has an unfavorably large condition number, $K\left(A_{13}\right)=1246$. Moving to the upper right corners in Fig. 2a the conditioning is improving. For example, for ternary mixture $30 \%-20 \%-50 \%$ the condition number is about $K=80$.

In a similar way, the choice of appropriate wavelengths of light can be done. Let us consider the data obtained for the above-mentioned mixture of hydrocarbons with equal concentrations $(1: 1: 1)$ (see Table 1$)$. The computation of the condition number for the matrix $A_{12}$ from our data obtained with $\lambda_{1}=670 \mathrm{~nm}$ and $\lambda_{2}=$ 
Table 1. Dependence the derivatives of refractive index on $\lambda$ for $C_{12} H_{26}-I B B-T H N$ $(1: 1: 1)$.

\begin{tabular}{l|ccccccc}
\hline$\lambda,[\mathrm{nm}]$ & $405^{1}$ & $488^{2}$ & $514^{3}$ & $589^{4}$ & $633^{1}$ & $670^{4}$ & $925^{4}$ \\
\hline$\left(\frac{\partial n}{\partial C_{1}}\right)_{c_{2}}$ & -0.1431 & -0.1272 & -0.1253 & -0.1211 & -0.1201 & -0.1170 & -0.1130 \\
\hline$\left(\frac{\partial n}{\partial C_{2}}\right)_{c_{1}}$ & -0.0475 & -0.0488 & -0.0484 & -0.0478 & -0.0495 & -0.0470 & -0.0456 \\
\hline${ }^{1}$ From ref. [17] \\
${ }^{2} \mathrm{~S}$. Wiegand et al., Germany, private communication \\
${ }^{3} \mathrm{M}$. BouAli, Spain, private communication \\
${ }^{4}$ The authors of the paper
\end{tabular}

925nm gives $K\left(A_{12}\right) \approx 1455$ while the computation done for the pair of wavelengths $\lambda_{1}=405 \mathrm{~nm}$ and $\lambda_{2}=633 \mathrm{~nm}[17]$ gives $K \approx 28$. That means that using the latter pair of wavelengths is preferable from the point of view of the expected precision of results for this particular mixture. The authors of [17] noticed that they knew about change of the light absorption by this mixture at $\lambda_{1}=405$.

The recommendation formulated above has a clear reason. As shown in Fig. 1, the derivative $\left(\partial n / \partial C_{2}\right)_{C 1}$ is almost independent of the wavelength. In the interval 670 $\mathrm{nm}<\lambda<925 \mathrm{~nm}$, the change of the derivative $\left(\partial n / \partial C_{1}\right)_{C 2}$ is very small, therefore the rows of the matrix $A_{12}$ almost coincide, and the matrix is ill-conditioned. In the interval $405 \mathrm{~nm}<\lambda<603 \mathrm{~nm}$, the change of $\left(\partial n / \partial C_{1}\right)_{C 2}$ is much larger, which leads to an essential decrease of the condition number and improvement of the computational accuracy.

It should be noted also that the value of the condition number of an illconditioned matrix is sensitive to the precision of the measurement of its elements. Fig. $2 \mathrm{~b}$ shows the dependence of the condition numbers of the matrices $A_{12}$ and $A_{13}$ and on the number of digits after comma for matrix elements. One can see that a reasonably good estimate of the condition number needs knowing at least 4 digits of matrix elements. Thus, the contrast factors for such kind of problems must be measured with an accuracy of at least 4 digits after comma.

Recall that the condition numbers of the matrices $A_{12}, A_{23}$ and $A_{13}$ differ. For instance, for the system shown in Fig. 2 the conditions number at point $C_{i 0}=$ $33.33 \%$ are $K\left(A_{12}\right)=1455, K\left(A_{23}\right)=550, K\left(A_{13}\right)=1245$ but their minima and maxima have the same coordinates.

\section{Experimental}

Hereafter we analyze the parameter space of contrast factors, $\left(\partial n / \partial C_{i}\right)$, for aqueous ternary mixtures. There exists a large body of experimental studies of thermodiffusion in aqueous solutions in binary mixtures. On the theoretical side the number of publications is much smaller but they exist. For example, the authors [36], [37] have found reasonable agreement between their simulations and experimental results for four binary aqueous mixtures. Studies in ternary mixtures are in an early stage.

The contrast factors were determined from the measured values of the refractive indexes $n$ for two different wave lengths. The refractive index was measured with the Abbe refractometer manufactured by Bellingham and Stanley model 60/LR with four and a half digits resolution. This refractometer does not include a light source. Two laser diodes, $670 \mathrm{~nm}$ and $925 \mathrm{~nm}$, were purchased from another provider. The laser beams were alternatively directed into refractometer through the opti- 


\section{ebruary 28, 2011

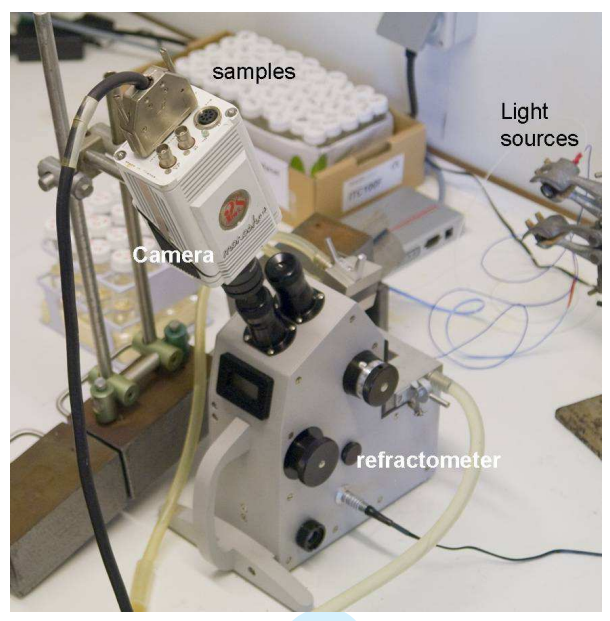

(a) Experimental setup

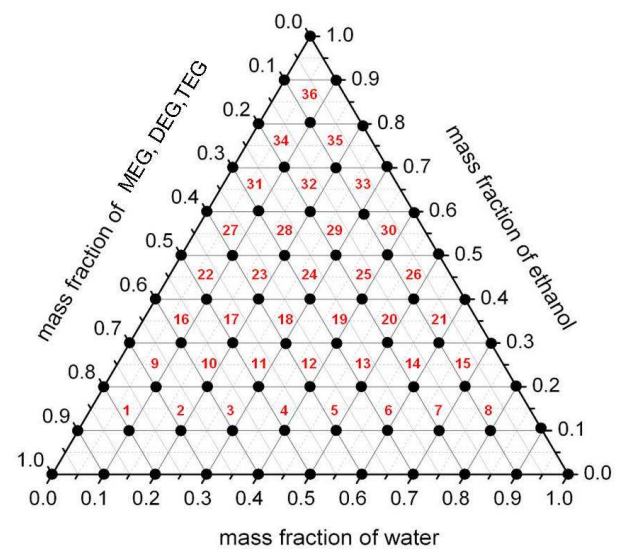

(b) Full coverage of a mixture with $10 \%$ sampling rate

Figure 3. Measurement of refractive index in ternary mixtures.

cal window. As infrared light is invisible, the human eye was replaced by a high sensitivity camera, see the photo of the setup in Fig. 3a. The crucial point for accurate measurements is the temperature stability. The constant temperature inside the instrument was maintained by water circulation through the thermostatically controlled High Precision Refrigerated Circulating Bath NESLAB RTE-300. The bath temperature was monitored to $\pm 0.01 \mathrm{~K}$ with a calibrated thermometer. Besides, all the measurements were conducted in a small room with well controlled air temperature.

Water (pure, deionized reagent Grade 3, 99.999\%), Ethanol (absolute, extra dry, 99.5\%), Monoethylene glycol (for analysis, 99.8\%) and Triethylene glycol, (for analysis, 99\%), t-butanol (for analysis ACS) were purchased from Acros Organics. Diethylene glycol (for analysis, 99\%) was purchased from Thermo Fisher Scientific. Dimethyl sulfoxide (> 99.9\%) was purchased from Sigma-Aldrich Chemie (courtesy S. Wiegand). Sixty six compositions (36 ternary +30 binary) should be studied to cover the whole range of concentrations for one ternary mixture with $10 \%$ sampling, see Fig. 3b. For the refractive index measurements $10 \mathrm{~g}$ of each mixture was prepared from these high quality products in a narrow-distributed concentration around rhe values of interest. An electronic balance manufactured by Sartorius with an accuracy of $10^{-2} \mathrm{mg} / 30 \mathrm{~g}$ was used. Before sample preparation the pure ingredients were placed in smaller bottles to prevent evaporation and/or pollution of basic solvents. The mixtures of a prescribed mass fraction were prepared directly on the weighing balance by adding ingredients drop by drop with a Hamilton syringe. At the beginning 66 bottles of the investigated ternary mixture were filled with a less volatile component and carefully closed by a cover with Teflon sealing. Small, but important point is a sample classification. The weight of each ingredient $m_{C_{i 0}}$ was adjusted in such a way that it was not larger than $m_{C_{i 0}} \pm 10^{-3} g$ in order to generate concentration $C_{i 0}$ very close to the values defined in the concentration matrix shown in Fig. 3b. The weights of all components were measured with an accuracy of $5 \cdot 10^{-5} \mathrm{~g}$. This procedure enables calculation of derivatives in concentration space with high precision, e.g. along the line with one really constant concentration. 


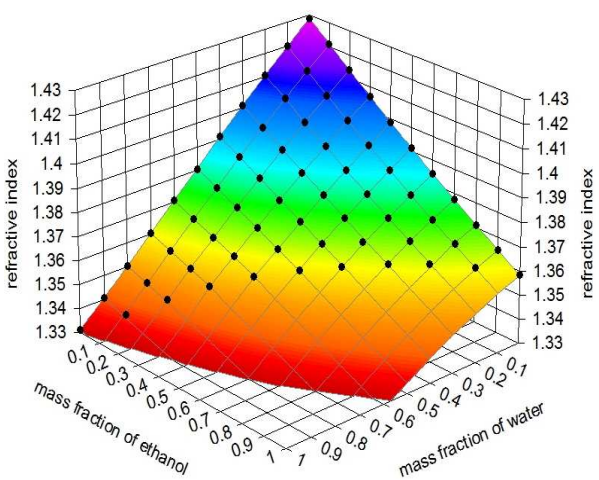

(a)

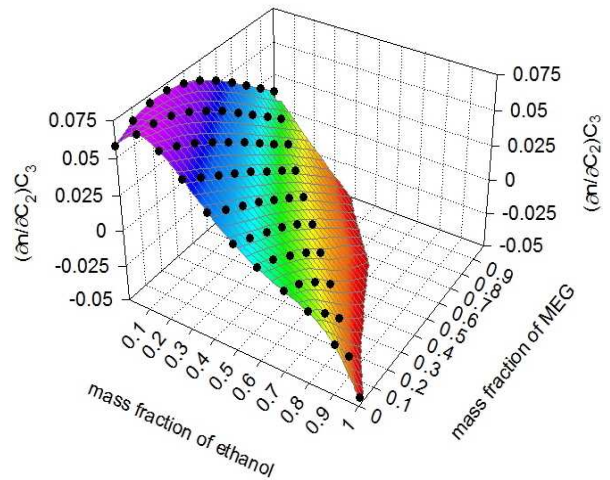

(b)

Figure 4. Water-Ethanol-MEG: (a) The fitting function $n_{1 f}\left(C_{1}, C_{2}\right)$ and (b) derivative $\partial n_{2 f}\left(C_{2}, C_{3}\right) / \partial C_{2}$ when $\lambda=670 \mathrm{~nm}$.

\section{Results}

\subsection{Methodology of results processing}

There exists a body of publications devoted to the prediction (calculation) of the refractive index (RI) on the basis of thermodynamic quantities. The most widely used is a mixing rule, when RI at a certain composition is calculated as a linear combination of the refractive indexes for the pure components [34], [35]. Our measurements for aqueous solutions did not confirm this tendency. Actually it is known, that the behavior of aqueous solutions is far from an ideal mixture. Thus for the use of multi-wavelength optical methods it is necessary to measure the refractive indices for each $\lambda$.

Continuous 3D surfaces in the form $n_{1 f}=n_{f}\left(C_{1}, C_{2}\right), n_{2 f}=n_{f}\left(C_{2}, C_{3}\right), n_{3 f}=$ $n_{f}\left(C_{1}, C_{3},\right)$ were constructed on the basis of the measured points. The aim of approximation is to fit the set of data points as closely as possible for a specified function $n_{f}$. The smoothness of the surface is important as the final target is the calculation of the derivatives with a good accuracy. All fits were tested graphically before accepting them as satisfactory, otherwise unwanted fluctuations between points may go undetected. Along with the condition to provide a minimal residual at the measured points an additional requirement was imposed: the derivatives $d n_{f} / d C_{i}$ on the boundaries of the triangle in Fig. $3 \mathrm{~b}$ must coincide with the contrast factors of binary mixtures, which are well known (at least for water-ethanol [33], [38]). Numerous options were tested for fitting (Chebyshev functions, regression) and we drew a conclusion that the best is the polynomial fitting. The polynomial fit for $n_{1 f}\left(C_{1}, C_{2}\right)$ was chosen as

$$
n_{1 f}\left(C_{1}, C_{2}\right)=\sum_{i, j=0}^{i, j=5} a_{i j} C_{1}^{i} C_{2}^{j}
$$

where the coefficients $a_{i j}$ depend on the concentrations set under consideration and they were sought by the non-linear regression method. The polynomials for other functions were constructed in a similar way. The maximum residual for both functions $n_{1 f}$ and $n_{2 f}$ does not exceed $0.04 \%$.

An example of the fitting surface $n_{1 f}$ built on the basis of 66 measured points with visible light $(\lambda=670 \mathrm{~nm})$ for the mixture Water-Ethanol-MEG is shown in Fig. 4a. The surface of the derivatives of the function $n_{2 f}$ for the same mixture, $\partial n_{2 f} / \partial C_{2}$, is shown in Fig. 4b. On the front edge one can easily identify a typical 


\section{Page 11 of 18 \\ 17:40

profile $(d n / d C)$ for water-ethanol binary mixture. Only the parts of the surfaces shown in Fig. 4 where $0 \leq C_{3}=1-C_{1}-C_{2} \leq 1$ have a physical sense.

It can be easily shown that the sum of the three derivatives inside the triangles (Figs. 5-7) obtained for each wavelength is equal to zero,

$$
\Sigma=\left(\frac{\partial n}{\partial C_{1}}\right)_{C_{2}}+\left(\frac{\partial n}{\partial C_{2}}\right)_{C_{3}}+\left(\frac{\partial n}{\partial C_{3}}\right)_{C_{1}}=0
$$

It is a simple but severe test for correctness of the fitting functions in Eq. (16). For the fields shown in Fig. $5, \Sigma \sim 10^{-12}$. The computed derivatives for each mixture form matrices (15) whose properties will be discussed in section IVb.

Here the attention is focused on the contrast factors of aqueous solutions containing glycols of different chain lengths: (water-ethanol) + Monoethylene Glycol (MEG); Diethylene Glycol (DEG) and Triethylene Glycol (TEG). These glycols are soluble in water in presence of Ethanol. The refractive indices for $\lambda=670 \mathrm{~nm}$ and $\lambda=925 \mathrm{~nm}$ were measured in 66 points shown in Fig. $3 \mathrm{~b}$ for MEG and DEG,

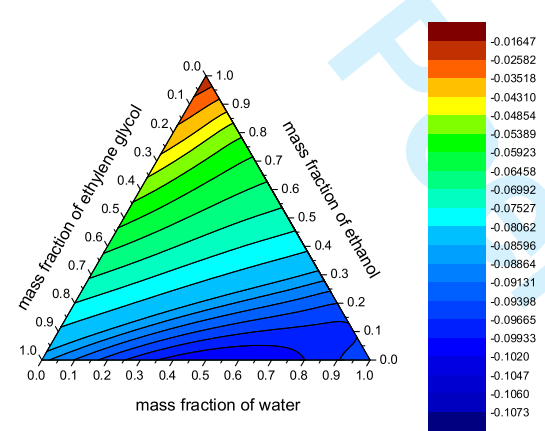

(a) $\lambda_{1}=670 \mathrm{~nm},\left(\partial n / \partial C_{1}\right)_{C 2}$

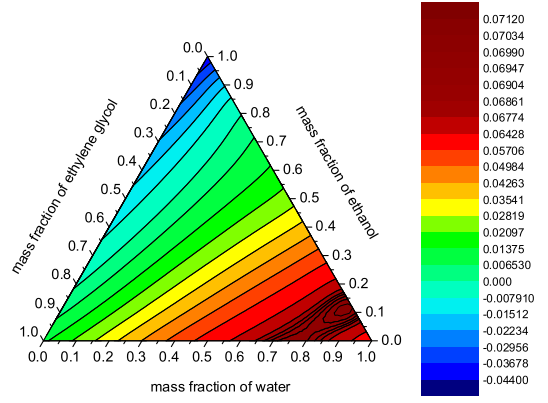

(c) $\lambda_{1}=670 \mathrm{~nm},\left(\partial n / \partial C_{2}\right)_{C 3}$

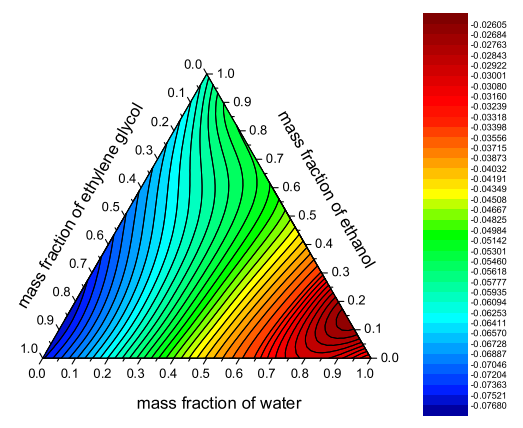

(e) $\lambda_{1}=670 \mathrm{~nm},\left(\partial n / \partial C_{3}\right)_{C 1}$

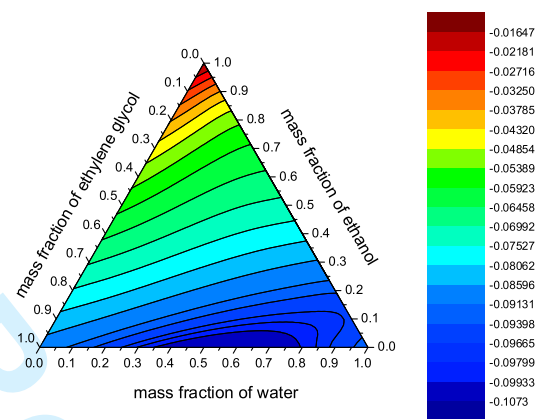

(b) $\lambda_{2}=925 \mathrm{~nm},\left(\partial n / \partial C_{1}\right)_{C 2}$

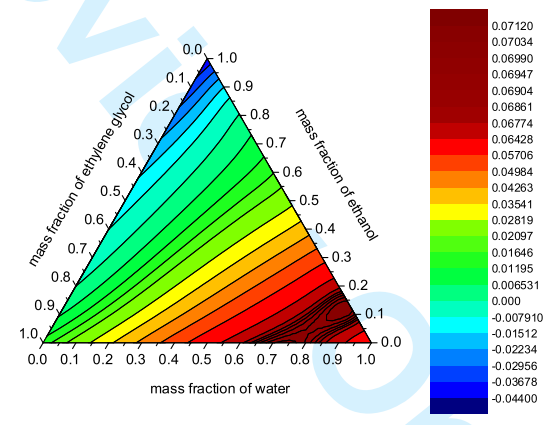

(d) $\lambda_{2}=925 \mathrm{~nm},\left(\partial n / \partial C_{2}\right)_{C 3}$

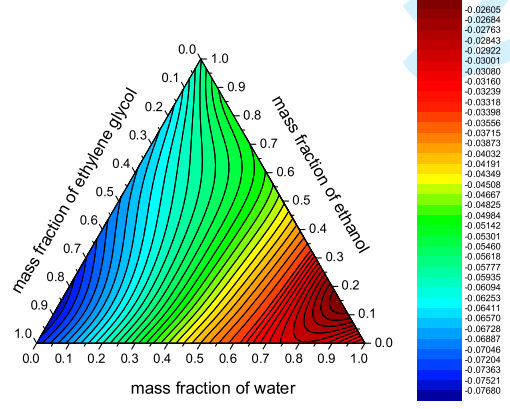

(f) $\lambda_{2}=925 \mathrm{~nm},\left(\partial n / \partial C_{3}\right)_{C 1}$

Figure 5. Contrast factors in Water-Ethanol-MEG obtained on the basis of 66 refractive index measurements for each $\lambda$ at $25^{\circ} \mathrm{C}$. 


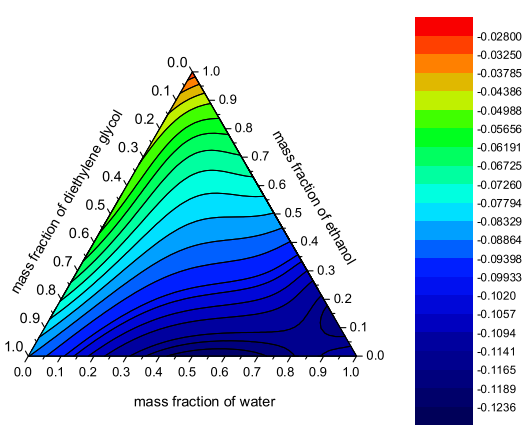

(a) $\lambda_{1}=670 \mathrm{~nm},\left(\partial n / \partial C_{1}\right)_{C 2}$

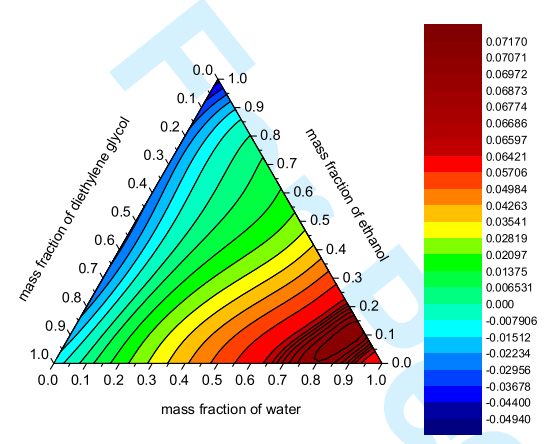

(c) $\lambda_{1}=670 \mathrm{~nm},\left(\partial n / \partial C_{2}\right)_{C 3}$

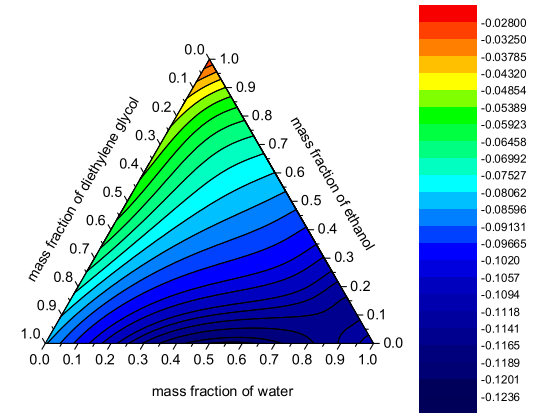

(b) $\lambda_{2}=925 \mathrm{~nm},\left(\partial n / \partial C_{1}\right)_{C 2}$

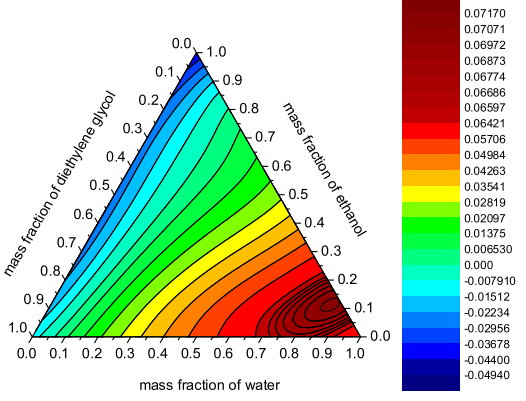

(d) $\quad \lambda_{2}=925 \mathrm{~nm},\left(\partial n / \partial C_{2}\right)_{C 3}$

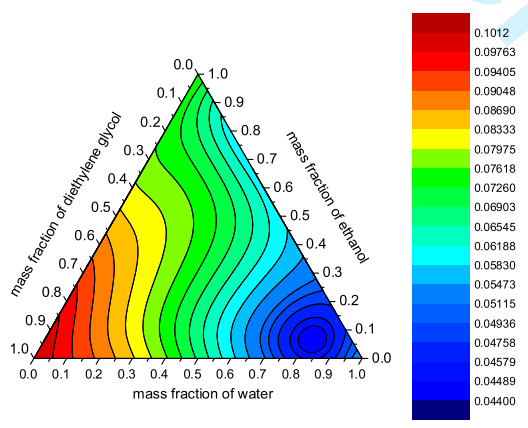

(e) $\lambda_{1}=670 \mathrm{~nm},\left(\partial n / \partial C_{3}\right)_{C 1}$

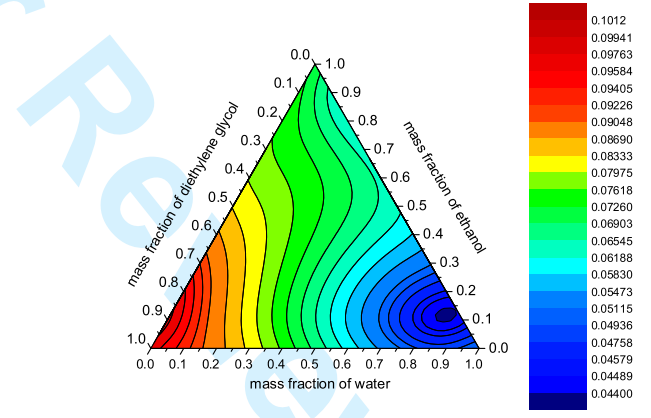

(f) $\lambda_{2}=925 \mathrm{~nm},\left(\partial n / \partial C_{3}\right)_{G 1}$

Figure 6. Contrast factors in Water-Ethanol-DEG obtained on the basis of 66 refractive index measurements for each $\lambda$ at $25^{\circ} \mathrm{C}$.

and in 44 points for TEG (20\% sampling rate). In addition we have conducted RI measurements (35 points) in water rich region for the mixture Water-t-butanoldimethyl sulfoxide (DMSO).

The results of the measurements for Water-Ethanol-MEG are summarized in Fig. 5 as isolines of the three derivatives $\left(\partial n / \partial C_{1}\right)_{C 2},\left(\partial n / \partial C_{2}\right)_{C 3}$, and $\left(\partial n / \partial C_{3}\right)_{C 1}$. The plots on the left side correspond to $\lambda_{1}=670 \mathrm{~nm}$ while plots on the right side correspond to $\lambda_{2}=925 \mathrm{~nm}$. The isolines are not parallel inside all the triangles indicating that the mixture is not thermodynamically ideal. So, the contrast factors can not be accurately determined from those of pure components using a variety of different mixing rules. The entire field of the derivatives at constant $C_{2}$ (ethanol) has a negative sign (see Fig. 5a) while the derivatives at constant $C_{1}$ (water) are positive, see Fig. 5e. The derivatives at constant $C_{3}$ (MEG) are sign-changing, and it is reasonable. At $C_{3}=$ const $=0$ the mixture transforms into a binary water-ethanol solution whose well studied contrast factor is sign-changing with the concentration [33]. As follows from Fig. 5 the structure of isolines for different $\lambda$ but identical derivatives is rather similar, that means that 


\section{Page 13 of 18

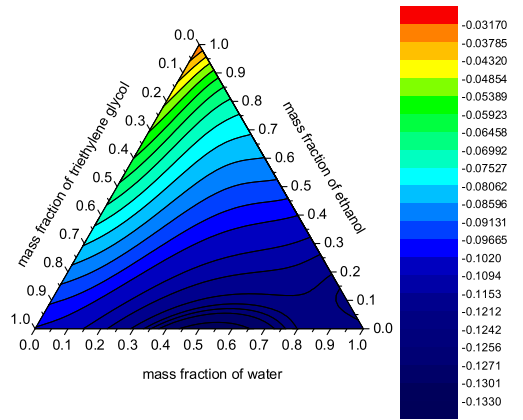

(a) $\lambda_{1}=670 \mathrm{~nm},\left(\partial n / \partial C_{1}\right)_{C 2}$

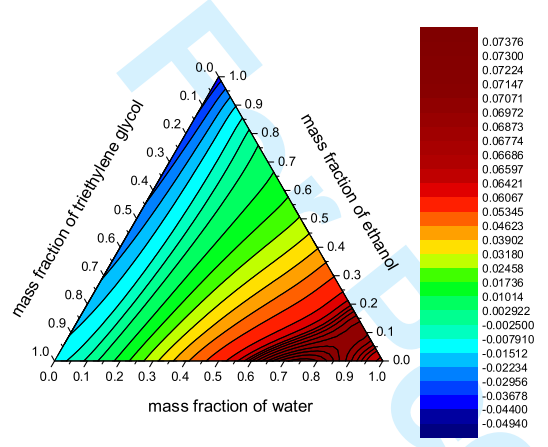

(c) $\lambda_{1}=670 \mathrm{~nm},\left(\partial n / \partial C_{2}\right)_{C 3}$

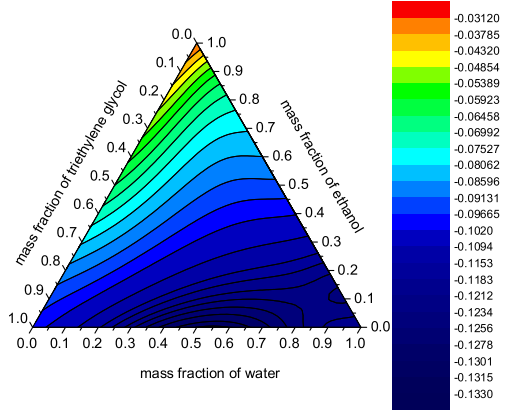

(b) $\lambda_{2}=925 \mathrm{~nm},\left(\partial n / \partial C_{1}\right)_{C 2}$

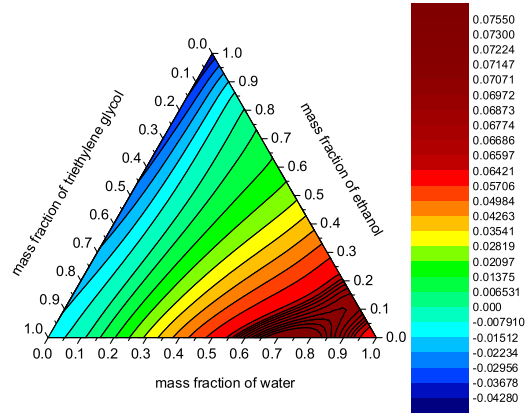

(d) $\quad \lambda_{2}=925 \mathrm{~nm},\left(\partial n / \partial C_{2}\right)_{C 3}$

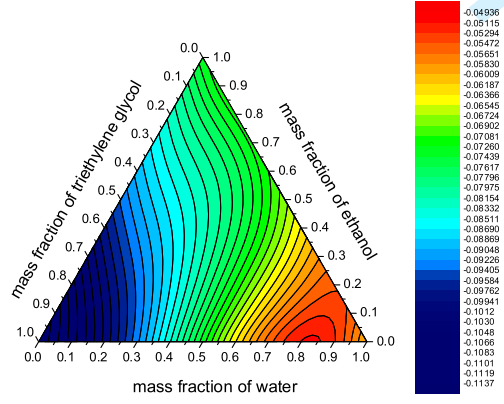

(e) $\lambda_{1}=670 \mathrm{~nm},\left(\partial n / \partial C_{3}\right)_{C 1}$

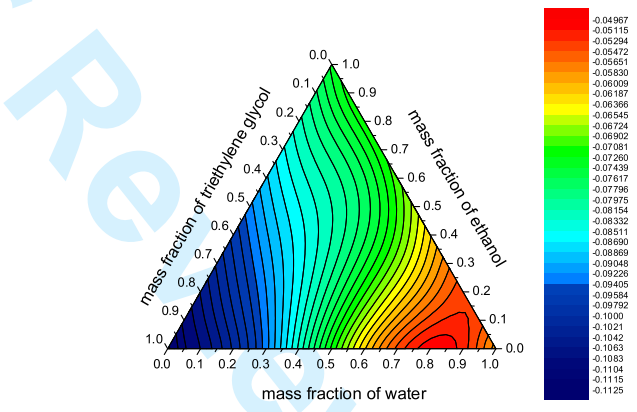

(f) $\lambda_{2}=925 \mathrm{~nm},\left(\partial n / \partial C_{3}\right)_{C 1}$

Figure 7. Contrast factors in Water-Ethanol-TEG obtained on the basis of 41 refractive index measurements for each $\lambda$ at $25^{\circ} \mathrm{C}$.

the raws in matrices (15) will be quasi-proportional.

The results of the measurements for Water-Ethanol-DEG mixture are summarized in Fig. 6 as isolines of the all the three derivatives $\left(\partial n / \partial C_{1}\right)_{C 2},\left(\partial n / \partial C_{2}\right)_{C 3}$, and $\left(\partial n / \partial C_{3}\right)_{C 1}$. The plots on the left side correspond to $\lambda_{1}=670 \mathrm{~nm}$ while plots on the right side correspond to $\lambda_{2}=925 \mathrm{~nm}$. The curvature of isolines is much stronger pronounced than in the case of MEG and it indicates larger deviations from ideal refractive behaviour. Perhaps, it is related to the point that the chemical structure of DEG has longer chain than MEG. The sign of the derivatives is similar to that of MEG: negative at constant $C_{2}$ (ethanol); positive at constant $C_{1}$ (water) and sign-changing at constant $C_{3}$ (MEG). The behavior of various derivatives in concentration space is very different; $\left(\partial n / \partial C_{1}\right)_{C 2}$ attains deep minima at water rich side while $\left(\partial n / \partial C_{2}\right)_{C 3}$ has maximum at this region; $\left(\partial n / \partial C_{3}\right)_{C 1}$ attains maximum at the water poor region (left bottom corners in Fig. 6e) and diminishes with the decrease of water concentration. From a short look the structure of the isolines for identical derivatives but different $\lambda$ is rather dissimilar and, correspondingly, the invertibility of matrices (15) could be better than in the case of MEG. 


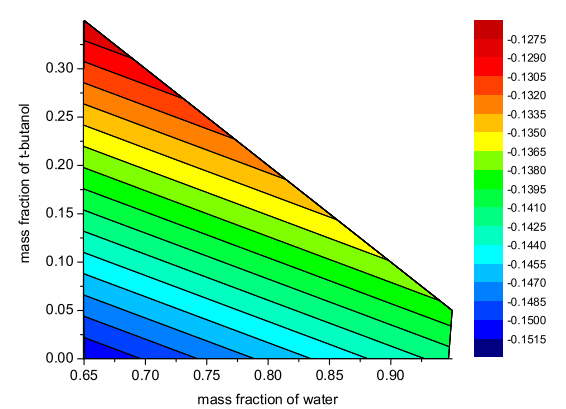

(a) $\lambda_{1}=670 \mathrm{~nm},\left(\partial n / \partial C_{1}\right)_{C 2}$

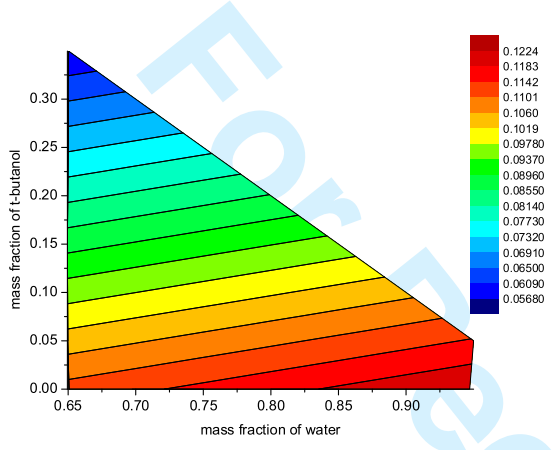

(c) $\lambda_{1}=670 \mathrm{~nm},\left(\partial n / \partial C_{2}\right)_{C 3}$

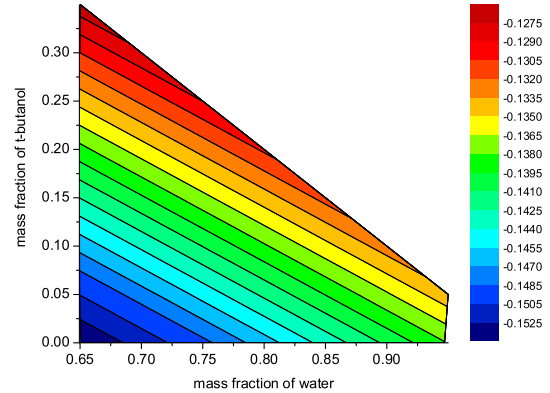

(b) $\lambda_{1}=925 \mathrm{~nm},\left(\partial n / \partial C_{1}\right)_{C 2}$

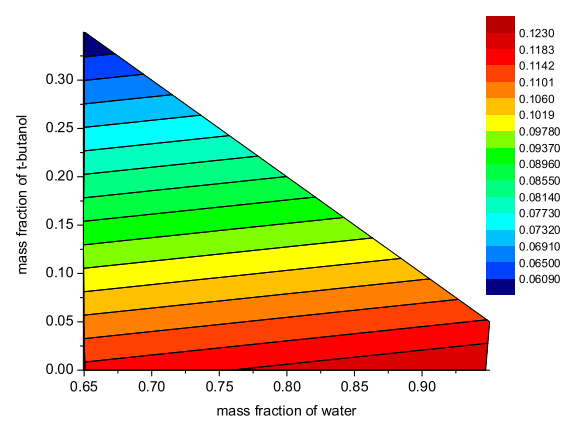

(d) $\lambda_{1}=925 \mathrm{~nm},\left(\partial n / \partial C_{2}\right)_{C 3}$

Figure 8. Measured contrast factors in Water-t-butanol-DMSO on the basis of 35 refractive index measurements at $25^{\circ} \mathrm{C}$.

The results of the measurements for Water-Ethanol-TEG are summarized in Fig. 7 also as isolines of the three derivatives $\left(\partial n / \partial C_{1}\right)_{C 2},\left(\partial n / \partial C_{2}\right)_{C 3}$, and $\left(\partial n / \partial C_{3}\right)_{C 1}$ for the same pair of the wavelengths. The structure of isolines is less wavy than in the case of DEG and slightly resembles the structure of the isolines for MEG. The sign of the derivatives is similar to that of MEG and DEG: negative at constant $C_{2}$ (ethanol); positive at constant $C_{1}$ (water) and sign-changing at constant $C_{3}$ (glycol).

The absolute maximum values of all the derivatives as well as the amplitudes of any derivatives in each triangle grow by the replacement MEG $\rightarrow$ DEG $\rightarrow$ TEG. For all the mixtures the largest amplitude of the derivatives occurs at constant $C_{2}$ (ethanol) and the smallest one at constant $C_{3}$ (glycol).

The results of the measurements for aqueous solutions which does not contain glycol are shown in Fig. 8 as isolines of the two derivatives $\left(\partial n / \partial C_{1}\right)_{C 2},\left(\partial n / \partial C_{2}\right)_{C 3}$ for the wavelengths in red $\lambda_{1}=670 \mathrm{~nm}$ and infrared $\lambda_{2}=925 \mathrm{~nm}$ regions. For this mixture (Water-t-butanol-DMSO) we have studied only water rich region with $5 \%$ sampling, $0.65<C_{2}<0.95$. In the considered region of compositions the isolines of the derivatives are parallel, indicating an ideal refractive behaviour. The derivatives at constant $C_{3}$ (DMSO) have practically the same slopes for both $\lambda$ (compare Fig. 8c and Fig. 8d. However the derivatives at constant $C_{2}$ (t-butanol) have different slopes enabling the possibility to invert matrices (15).

\subsection{Feasibility study for aqueous solutions}

Now, the mathematical approach described in section II.C and detailed in Appendix $\mathrm{B}$ is applied for the contrast factors determined in the previous paragraph.

The condition number $K$ has been calculated in the norm $l_{2}$ Eq. (22) for the matrices $A_{13}$ found for each ternary mixture. The results are presented in Fig. 9. The darker (lighter) regions correspond to lower (higher) values of $K$ and, correspond- 


\section{Page 15 of 18 Philosophical Magazine \& Philosophical Magazine Letters}

1

2

3

4

5

6

7

8

9

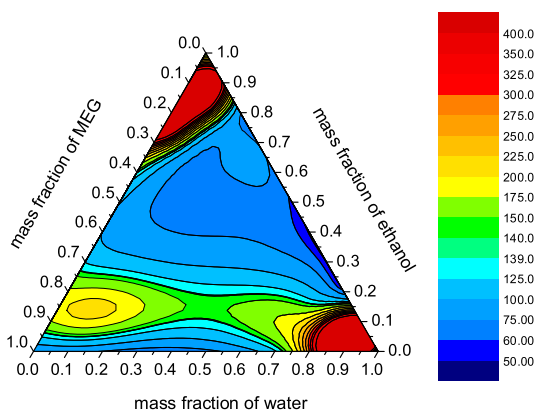

(a) Water-Ethanol-MEG

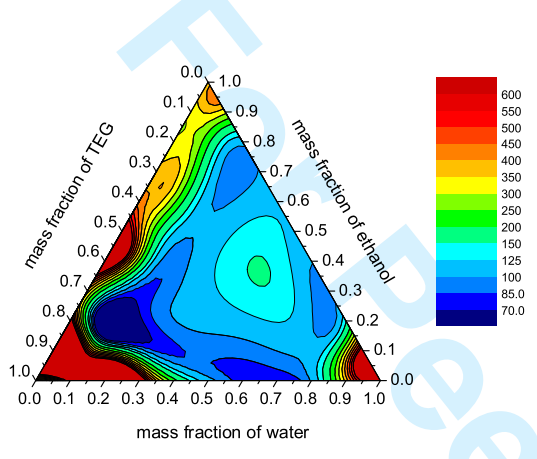

(c) Water-Ethanol-TEG

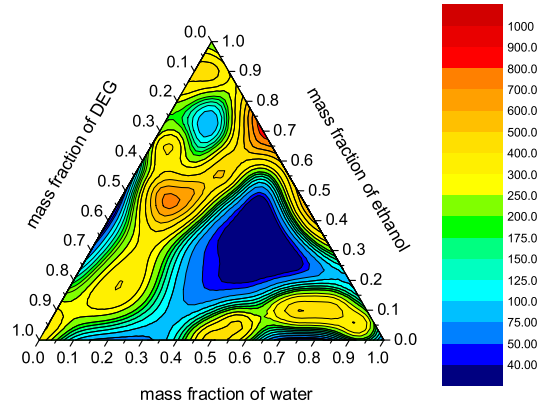

(b) Water-Ethanol-DEG

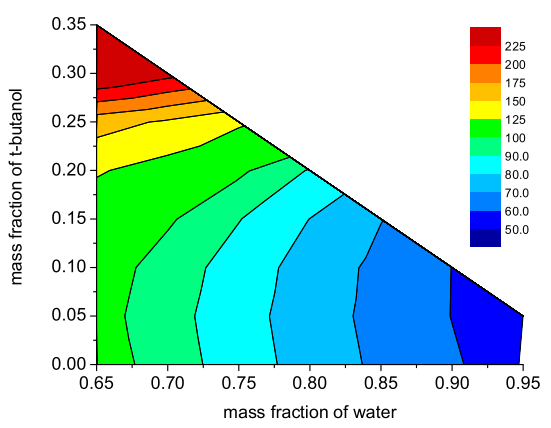

(d) Water-t-butanol-DMSO

Figure 9. Isolines of conditions number $K$ of matrices $A_{12}$ when $\lambda_{1}=670 \mathrm{~nm}$ and $\lambda_{2}=925 \mathrm{~nm}$. Regions in dark blue colors correspond to smaller $K$, i.e. smaller loss of precision introduced by the solution of Eqs. (9)-(10).

ingly, a smaller (larger) relative error. Let us discuss first the mixtures containing water and ethanol, Figs. 9(a-c). All the fields have multiple maxima (unfavourable compositions) and minima (favourable compositions) in the triangles of concentrations. For each field, the landscape of $K$ has a rather quaint shape, which cannot be predicted without calculations. The details of the obtained landscapes can be used for an optimal choice of the compositions to be chosen for measurements. Typically, the condition number is smaller in the middle of the triangle and larger near the angles of the triangle, but there exist also some islands of unfavourable compositions. As predicted in the previous subsection, the mixture Water-Ethanol-DEG has a deepest minimum of $K$ (about 40) and hence it is an optimal choice for carrying out measurements among the three mixtures. Note, that the minima of $K$ for other matrices are smaller, i.e. $K_{\min }=100$ for $A_{12}$ and $K_{\min }=66$ for $A_{23}$. The minimum values of $K$ for the mixture Water-Ethanol-TEG are slightly larger than that for other mixtures.

Fig. 9(d) contains results obtained for the system Water-t-butanol-DMSO. The characteristic values of $K$ for that mixture are lower than those for the mixtures discussed above, especially in the region of relatively small concentrations of $\mathrm{t}$ butanol and DMSO. Thus, when the light wavelengths are fixed as $\lambda_{1}=670 \mathrm{~nm}$ and $\lambda_{2}=925 \mathrm{~nm}$, the latter mixture and mixture water-ethanol-DEG seems to be the best choice for the application of two-wavelength optical methods.

\section{Conclusions}

The application of two-wavelength optical methods (OBD and ODI) for the determination of Soret coefficient is considered. It has been shown that the methods are feasible only for definite pairs of light wavelength and for definite compositions of 
mixtures, because the accuracy of the methods can be strongly diminished if the matrices of contrast factors are ill-conditioned. For example, the successful application of wavelengths $\lambda_{1}=408 \mathrm{~nm}$ and $\lambda_{2}=633 \mathrm{~nm}$ for Fontainebleau mixtures in [17] does not provide guarantee that it will be equally successful for another compositions and/or for another liquids. The preliminary analysis for each chosen mixture and pair of wavelength is unavoidable.

A simple approach for studying the feasibility of the methods is suggested, which is based on the measurement of the refractive indices $n_{i}\left(C_{1}, C_{2}\right), i=1,2$ for two wavelengths on a dense mesh, a polynomial interpolation of fields $n_{i}\left(C_{1}, C_{2}\right)$, a computation of the matrices of contrast factors, and calculation of the condition numbers of these matrices. We have performed 470 measurements of the refractive index in aqueous solutions, which includes preparation of 235 different samples and measurements in two wave lengths $\lambda_{1}=670 \mathrm{~nm}$ and $\lambda_{2}=925 \mathrm{~nm}$. The developed approach has been applied to the examined ternary mixtures (water-ethanolMEG; water-ethanol-DEG; water-ethanol-TEG; Water-t-butanol-DMSO), and the regions of feasibility /infeasibility of the methods have been revealed.

\section{Acknowledgments}

This work is supported by the PRODEX programme of the Belgian Federal Science Policy Office and ESA. The authors are indebted to V. Yasnou (ULB, Brussels) for help in conducting of the experiments and Dr. A.Mialdun (ULB, Brussels) for useful discussions.

\section{Appendix A: An example demonstrating the essence of the problem with ill-conditioned matrix.}

Let us consider the following toy example. Assume that exact system (11) is

$$
\begin{aligned}
\Delta C_{1}+\Delta C_{2} & =2, \\
\Delta C_{1}+1.01 \Delta C_{2} & =2.01,
\end{aligned}
$$

with an obvious solution $\Delta C_{1}=\Delta C_{2}=1$. The condition number of the matrix based on norm $l_{2}$ is about $K \approx 400$. Assume now that the measurements of the right-hand side terms $F_{1}, F_{2}$ are done with the precision of $0.1 \%$, and the system of equations based on the measured values of $\vec{F}$ is

$$
\begin{aligned}
\Delta C_{1}+\Delta C_{2} & =2.002, \\
\Delta C_{1}+1.01 \Delta C_{2} & =2.008 .
\end{aligned}
$$

The solution of the latter system is $\Delta C_{1}=1.402, \Delta C_{2}=0.6$, i.e., the relative error of the obtained values of $\Delta C_{1}$ and $\Delta C_{2}$ is about $40 \%$. That means that allegedly good precision of $0.1 \%$ in the measurements of $F_{i}$ is actually insufficient. Obviously, before carrying out the measurements of $\vec{F}$, it is necessary to estimate the possible loss of precision by means of the analysis of matrix $A$ which is measured in advance.

\section{Appendix B: Definition and calculation of condition number}

Here we present some definitions and algorithms for calculation of the condition number in different norms [39]. 


\section{$\begin{array}{crr}\text { ebruary 28, 2011 } & 17: 40 & \text { Philosophical Magazine Ternary } \\ \text { Page 17 of } \mathbf{1 8} & \text { Philosophical Magazine \& Philosophical Magazine Letters }\end{array}$}

Philosophical Magazine

Let us consider a linear system of equations

$$
A \vec{X}=\vec{F}
$$

its solution is $\vec{X}=A^{-1} \vec{F}$. Assume that the vector of data in the right-hand side of (19) is measured with a certain error $\vec{f}$ (i.e., the measured data vector is $\vec{F}+\vec{f}$ rather than $\vec{F}$ ), which leads to an error $\vec{x}$ in the solution, $\vec{x}=A^{-1} \vec{f}$. The relative error of the measurement data can be defined as $\delta(\vec{F})=\|\vec{f}\| /\|\vec{F}\|$, where \|\| means a certain norm of the vector (see (12)-(14)), while the relative error of the calculated vector $\vec{X}$ is $\delta(\vec{X})=\|\vec{x}\| /\|\vec{X}\|=\left\|A^{-1} \vec{f}\right\| /\left\|A^{-1} \vec{F}\right\|$.

The condition number of matrix $A$ is defined as

$$
K(A)=\max \frac{\delta(\vec{X})}{\delta(\vec{F})}=\max \frac{\left\|A^{-1} \vec{f}\right\| \cdot\|\vec{F}\|}{\|\vec{f}\| \cdot\left\|A^{-1} \vec{F}\right\|}
$$

where maximum is taken over any vectors $\vec{F}$ and $\vec{f}$. This quantity shows to what extent the relative error $\delta(\vec{X})$ of the computed quantities can be enhanced in the course of computations, as compared to the relative error $\delta(\vec{F})$ of the measured data.

Let us define norms of matrices as

$$
\left\|A^{-1}\right\|=\max \frac{\left\|A^{-1} \vec{f}\right\|}{\|\vec{f}\|},\|A\|=\max \frac{\|F\|}{\left\|A^{-1} \vec{F}\right\|}
$$

then

$$
K(A)=\left\|A^{-1}\right\| \cdot\|A\| .
$$

When the vector norms $l_{1}$ (Eq. (12)) or $l_{\infty}$ (Eq. (14)) are used, the norms of matrices $m \times m$ are computed, correspondingly, as

$$
\|A\|_{1}=\max _{1 \leq j \leq m} \sum_{i=1}^{m}\left|A_{i j}\right| \quad \text { and } \quad\|A\|_{\infty}=\max _{1 \leq i \leq m} \sum_{j=1}^{m}\left|A_{i j}\right| .
$$

In the case of the norm $l_{2}$ (Eq.(13)), the condition number can be calculated as

$$
K(A)=\left(\left|q_{\max }\right| /\left|q_{\min }\right|\right)^{1 / 2}
$$

where $q_{\max }$ and $q_{\min }$ are the largest and the smallest eigenvalues of the matrix $A^{T} A\left(A^{T}\right.$ is a transpose of $A$ ). For a matrix $2 \times 2, A=\left(\begin{array}{ll}a & b \\ c & d\end{array}\right)$, the eigenvalues can be found as

$$
q_{\text {max }, \text { min }}=\frac{a^{2}+b^{2}+c^{2}+d^{2}}{2} \pm \frac{\sqrt{4(a b+c d)^{2}+\left[\left(a^{2}+c^{2}\right)-\left(b^{2}+d^{2}\right)\right]^{2}}}{2} .
$$

As an example, let us consider the matrix $A=A_{12}$ obtained from the measurements for the system $C_{12} H_{26}-I B B-T H N$ and the composition $(1: 1: 1)$ with the light wavelengths $\lambda_{1}=670 \mathrm{~nm}$ and $\lambda_{2}=925 \mathrm{~nm}$, 
$A=\left(\begin{array}{l}-0.11702-0.04697 \\ -0.11299-0.04557\end{array}\right)$ The determinant of that matrix is $\operatorname{Det}(A)=$

$-2.468 \cdot 10^{-5}$, hence

$A^{-1}=-0.4052 \cdot 10^{5}\left(\begin{array}{cc}-0.04557 & 0.04697 \\ 0.11299 & -0.11702\end{array}\right)$

Using the $l_{1}$ norm, we find

$$
\|A\|_{1}=\max \{|a|+|c|,|b|+|d|\}=\max \{0.2300,0.0925\}=0.23 .
$$

Similarly, we find $\left\|A^{-1}\right\|_{1}=1.6644 \cdot 10^{4}$, thus

$$
K=\|A\|_{1} \cdot\left\|A^{-1}\right\|_{1}=1528 .
$$

Calculation by means of formula (22) gives a slightly smaller value 1455 .

\section{References}

[1] J.S. Turner, Multicomponent convection. Annu. Rev. Fluid Mech. 17 (1985) p.11.

[2] K. Ghorayeb, A. Firoozabadi, and T. Anraku, SPE journal 8(2) (2003) p.114.

[3] F. Montel, Entropie. 86 (1994) p.184.

[4] F.M. Richter, N. Dauphas and F.-Z. Tenga, Chemical Geology. 258 (2009) p.92.

[5] K. Harstad and J. Bellan, Int. J. Multiphase Flow. 26 (2000) p.1675.

[6] S. Van Vaerenbergh, S.R. Coriell, G.B. McFadden, B.T. Murray, J.C. Legros, J. Crystal Growth. 147 (1995) p.207.

[7] A. Firoozabadi, P. Cheng, AIChE J. 56(6) (2010) p.1398.

[8] R.M. Schmitt, Annual Rev. Fluid Mech. 26 (1994) p. 255.

[9] F.J. Bonner and L.O. Sundelöf, Z. Naturforsch. 39 (1984) p.656.

[10] A. Firoozabadi, K. Ghorayeb, K. Shukla, AIChE Journal, 46 (2000) p.892.

[11] L. J. T. M. A Kempers, J. Chem. Phys. 115 (2001) p.6330.

[12] M. El Maâtaoui, PhD Thesis, Univ. Paul Sabatier, Toulouse, France (1986)

[13] R. Kita, S. Wiegand, J. Luettmer-Strathmann, J. Chem. Phys. 121 (2004) p.3874.

[14] J.K. Platten, M.M. Bou-Ali, P. Costeseque, J. F. Dutrieux, W. Köhler, C. Leppla, S.Wiegand and G. Wittko, Phil. Mag. 83 (2003) p.1965.

[15] A.Leahy-Dios, M. M. Bou-Ali, J. K. Platten, A.J. Firoozabadi, J. Chem. Phys. 122 (2005) 234502.

[16] P. Blanco, M. M. Bou-Ali, J. K. Platten, D.A. de Mezquia, J. A. Madariaga and C. Santamara, J. Chem. Phys. 132 (2010) 114506.

[17] A. Königer, H. Wunderlich, and W. Köhler, J. Chem. Phys. 132 (2010) 174506.

[18] M. Giglio and A. Vendramini,Appl. Phys. Lett., 25 (1974) p.555.

[19] H. Cabrera, L. Martí-López, E. Sira, K. Rahn, and M. García-Sucre, J. Chem. Phys. 131, (2009) 031106.

[20] P. Kolodner and H. Williams and C. Moe, J. Chem. Phys. 88 (1988) p.6512.

[21] W. Köhler and P. Rossmanith, J. Phys. Chem. 99 (1995) p.5838.

[22] S. Wiegand, J. Phys. Condens. Matter, 16 (2004) p.357.

[23] V. Shevtsova, Adv. Space Research, 46 (2010) p.672.

[24] A. Mialdun and V. Shevtsova, Int. J. Heat Mass Transfer, 51 (2008) 3164.

[25] de Groot S. R. and Mazur P., Non-equilibrium Thermodynamics (North-Holland Publishing, Amsterdam) 1962.

[26] J.K. Platten and J.C. Legros, Convection in Liquids (Berlin: Springer) 1984.

[27] I.I. Ryzhkov and V. Shevtsova, Phys. Fluids. 19 (2007) 027101.

[28] M. Bou-Ali and J. K. Platten, J. Non-Equilib. Thermodyn. 30 (2005) 385.

[29] S. Van Vaerenbergh and J.C. Legros. Diffusion and thermodiffusion in microgravity, in Physics of Fluids in Microgravity (edited by R. Monti), 178 (Taylor \& Francis, London, 2001).

[30] K. B. Haugen and A. Firoozabadi, J. Phys. Chem. B 110 (2006) 17678.

[31] R. Piazza, Phil. Mag. 83 (2003) 2067.

[32] A. Mialdun and V. Shevtsova, Microgravity Sci. Technol., 21 (2009) p. 31.

[33] A. Königer, B. Meier, and W. Köhler, Phil. Mag. 89, (2009) p.907.

[34] Z. Atik, J. Chem. Thermodyn. 38 (2006) p.201.

[35] J. V. Herráez and R. Belda, J. Solution Chem. 35 (2006) p.1315.

[36] C., Nieto-Draghi, J.B. Avalos, and B. Rousseau, J. Chem. Phys. 122 (2005) 114503.

[37] Rousseau, B., C. Nieto-Draghi, and J.B. Avalos, The role of molecular interactions in the change of sign of the Soret coefficient. Europhys. Lett. 67 (2004) p. 976.

[38] P. Kolodner, H. Williams, and C.Moe, Optical measurement of the Soret coefficient of ethanol/water solutions J. Chem. Phys. 88 (1988) p.6512.

[39] N. Trefethen and D. Bau, Numerical Linear Algebra (SIAM, 1997). 